$<0.05$, fold-change greater than 2 -fold and average normalised expression greater than 3. Five upregulated DEGs with the highest logarithmic fold change and 2 down regulated genes were validated using quantitative real-time polymerase chain reaction (RT-PCR). Protein expression of significantly DEG was evaluated with immunohistochemistry (IHC) in tumour tissue, peritumour adipose tissue and distal adipose tissue slides.

Results A total of $64 / 770$ DEGs were identified in peritumoral adipose tissue compared to distal adipose tissue. Up-regulated genes (28.1\%) included COL1A1, SFRP2, FGF7, PLA2G2A, NGFR and SFRP2. Down regulated genes (71.9\%) included LEF1 and CDH1. Differential expression was validated with qRT-PCR (COL1A1 p=0.007; SFRP2 p=0.057; FGF7 ns; PLA2G2A ns; NGFR ns; LeF1 $\mathrm{p}=0.03$; CDH1 $\mathrm{p}=0.09$ ). IHC revealed differential distribution of COL1A1 showing maximum expression in tumor tissue decreasing at more distant sites.

Conclusion Our results have shown altered cancer associated pathways in peritumour stromal compartment, including down-regulation of Wingless/Integrated (WNT) pathway. Furthermore, we have identified significant up regulation of COL1A1 in peritumour adipose tissue compared to distal adipose tissue. COL1A1 may have a role in local invasion and distant metastasis, possibly mediated by SFRP2, a known procollagen c proteinase enhancer and WNT antagonist. SFRP2 and COL1A1 may represent potential stromal therapeutic targets in colon cancer.

\section{OWE-24 CT COLONOGRAPHY IN NATIONAL BOWEL SCREENING PROGRAM, IS IT REDUCING THE BURDEN?}

\footnotetext{
1,2Soad Elkady*, ${ }^{1}$ Andrew Muller. 'East Kent Hospitals University Foundation NHS Trust, Canterbury, Kent, UK; ${ }^{2}$ University of Alexandria, Egypt
}

10.1136/gutjnl-2019-BSGAbstracts.357

Introduction CT Colonography (CTC) or virtual colonoscopy offers an effective non-invasive technique to assess the bowel if conventional colonoscopy fails or is not done due to patients' factors.

Aim of the work To identify if CTC can actually help decrease the burden by decreasing the number and type of endoscopy required especially in patients with multiple comorbidity.

Methods We reviewed CTC requested for patients in the National bowel screening program over a period of 9 years from end of 2009 till beginning of 2018. Post CTC colonoscopy was reviewed and results assessed.

Results 220 CTC procedures were requested over the study period for 206 patients. Most patients (194) required one CTC, ten patients had it twice and two patients had it three times. Age of the patients at the time of the procedure ranged between 60 years to 81 years old. 60\% were female versus $40 \%$ male. Most common indications for the CTC were failed endoscopy in 56\%, patients with multiple comorbidities $15 \%$ and patient preference in $7 \%$ of cases.

Colonic pathology was reported in 68\% (149) of the cases, extra-colonic in 21\% (47) and no pathology identified in $10 \%$ (21) of the cases. Colonic pathology included colonic masses, polyps, stricture and diverticular disease. Important extracolonic findings reported included metastatic disease, abdominal aortic aneurysm, fatty liver, renal and liver cysts.

Only 61(28\%) cases had a post CTC endoscopy within maximally 1 year interval. Most were sigmoidoscopy 41(67\%), followed by colonoscopy 14 (23\%). Deep sedation was required for 5 cases (8\%); 3 were colonoscopy and 2 were sigmoidoscopy.

$15(7 \%)$ cases were reported by CTC as suggestive of colonic mass. Only 9 had a post CTC endoscopy; of which only 2 were found to have colonic mass. Colonic polyps and diverticular disease was found in 3 cases and no pathology identified in the remaining 3 .

$51(23 \%)$ cases were reported by CTC to have colonic polyps 41 had post CTC endoscopy of which 31 (76\%) were found to have polyps and polypectomy performed. One case (2\%) was found to have colonic mass. 5 (12\%) had only diverticular disease and one with telangiectasia and in 3 (7\%) cases no pathology was identified.

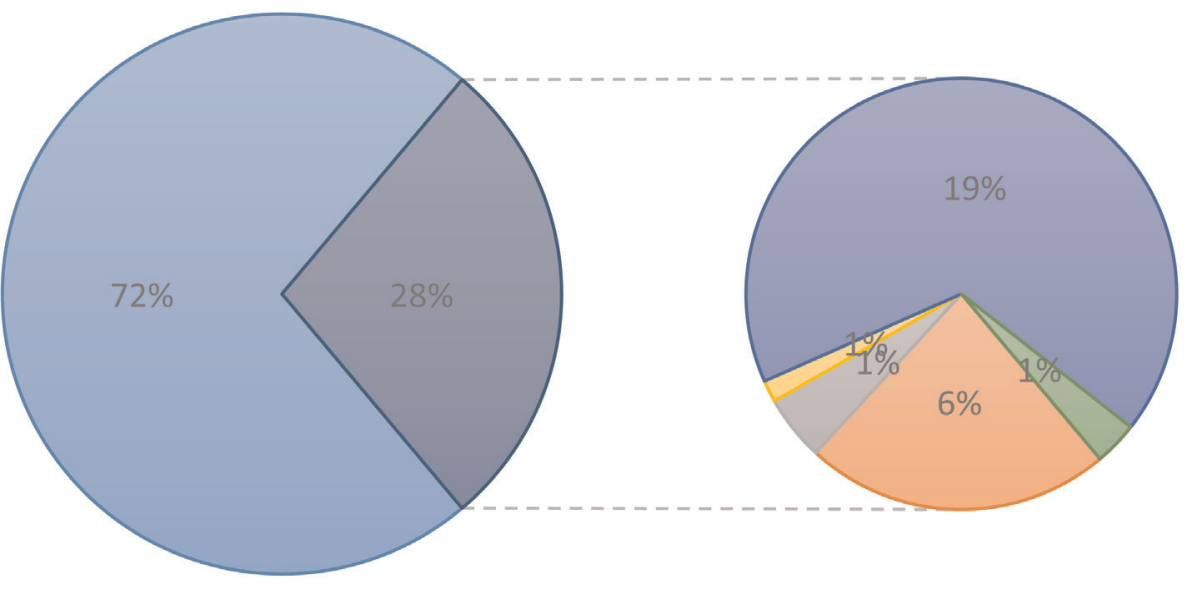

No

$\square$ colonoscopy under deep sedation colonoscopy

incomplete colonoscopy

sigmoidoscopy under deep sedation 
Conclusions CTC was useful guide to direct further management of the patients as regards any endoscopy being requested at all and type being mainly simple sigmoidoscopy in most of the cases. Combination of CTC with faecal occult blood in BSP could be a reasonable alternative for patient's assessment especially those elderly group with multiple comorbidities and challenging endoscopy.

\section{OWE-25 PATIENTS WITH MULTIPLE ADENOMAS IN BOWEL CANCER SCREENING PROGRAM ARE NOT REFERRED FOR GENETIC TESTING}

${ }^{1}$ James Alexander* ${ }^{2}$ Belinda Johnston, ${ }^{3}$ Tom Smith, ${ }^{3}$ Jonathan Fawkes, ${ }^{2}$ John Martin ${ }^{3}$ Edward Seward, 'Kevin Monahan. 'Chelsea and Westminster NHS Foundation Trust London, UK; ${ }^{2}$ Imperial College Healthcare NHS Foundation Trust, London, UK; ${ }^{3}$ University College London Hospitals NHS Foundation Trust, London, UK

\subsection{6/gutjn|-2019-BSGAbstracts.358}

Introduction Approximately one in twenty cases of colorectal cancer (CRC) are associated with germline mutations that confer higher susceptibility to the disease. Guidelines recommend that patients with ten or more adenomas be referred for genetic testing ${ }^{1}$, with evidence that suggests $>9 \%$ of these patients with 1-9 adenomas have a highly penetrant CRC predisposition syndrome ${ }^{2}$. The primary aim of this study was to quantify patients with ten or more adenomas in the West London (WL) and North Central London (NCL) Bowel Cancer Screening program (BCSP) centres. The secondary aim was to determine what proportion of these multiple adenoma patients were referred for genetic screening.

Methods A retrospective cross-sectional study was performed of patients who underwent colonoscopy following a positive faecal occult blood test $(\mathrm{FOBt})$ as part of the WL and NCL BCSPs between May 2007 \&amp June 2018. All polyps were examined histologically and only confirmed adenomas were included. Clinicopathological data including age and gender was recorded from BCSP patient records. Referrals to regional clinical genetics services were ascertained. Statistical analysis was performed in Graphpad Prism.

Results 11,337 patients underwent colonoscopy following positive FOBt and 5,650 (49.8\%) had 1 or more adenomas. 107 patients $(0.94 \%)$ had 10 or more adenomas. The proportion of patients with 10 or more adenomas was higher in NCL $\operatorname{BCSP}(1.1 \%)$ than in WL BCSP $(0.7 \%)(p=0.02 ; \chi 2$ test). 42 patients presented with 10 or more metachronous adenomas at the index colonoscopy or following an early follow up procedure; the remaining 65 patients undergoing excision of a total of 10 or more metachronous adenomas after subsequent surveillance colonoscopy. An accurate family history was not routinely collected in this population. Of the 107 patients with 10 or more adenomas, only $3(2.8 \%)$ were referred to a clinical genetics service.

Conclusions In two London BCSP centres, patients with ten or more metachronous adenomas are an uncommon finding. Despite guidelines advising genetic testing in this group, referral rates are low. A referral pathway and management strategies should be established to address this patient population.

\section{REFERENCES}

1. Hampel H, Bennett RL, Buchanan A, et al. A practice guideline from the American College of Medical Genetics and Genomics and the National Society of Genetic Counselors: referral indications for cancer predisposition assessment. Genet Med 2015:17:7-7.
2. Grover S, Kastrinos F, Steyerberg EW, et al. Prevalence and phenotypes of APC and MUTYH mutations in patients with multiple colorectal adenomas. Jama 2012;308:48-92.

\section{OWE-26 INFLAMMATORY RESPONSES IN PATIENTS WITH COLORECTAL CANCER AND IRON DEFICIENCY ANAEMIATREATED WITH ORAL IRON}

${ }^{1}$ Hafid Omar Al-hassi ${ }^{*},{ }^{2}$ Oliver Ng, ${ }^{3}$ Sian Faustini, ${ }^{1}$ Oliver Phipps, ${ }^{2,1}$ Edward Dickson, ${ }^{4}$ Manel Mangalika, ${ }^{4}$ Natalie Worton, ${ }^{4}$ Barrie Keeler, ${ }^{2}$ Austin Acheson, ${ }^{1,4}$ Matthew Brookes. 'University of Wolverhampton, Wolverhampton, UK; ${ }^{2}$ Nottingham Digestive Diseases Centre, University of Nottingham, Nottingham, UK; ${ }^{3}$ Institute of Immunology and Immunotherapy, University of Birmingham, Birmingham, UK; ${ }^{4}$ New Cross Hospital, Wolverhampton, UK

\subsection{6/gutjnl-2019-BSGAbstracts.359}

Introduction Colorectal cancer (CRC), a major cause of cancer-related mortality, is associated with iron deficiency anaemia (IDA) and is typically treated with oral iron. Iron is an essential nutrient element that is required for a diverse range of biological activities including the immune function (1). However, studies in animal and cell line models have shown that accumulation of iron in the gut can generate toxic reactive oxygen species and proinflammatory cytokines that results in tissue damage and CRC progression (2). We studied, for the first time in humans, the effect of oral (OI) versus intravenous iron (IVI) treatment on iron distribution, immune cell infiltration and systemic cytokine profile in CRC patients with IDA.

Methods Patients with CRC and IDA received oral-ferrous sulphate $(n=20)$ or intravenous ferric carboxymaltose $(n=20)$ for 2 weeks. Tissue was analysed for iron loading using Prussian-Blue staining. Tissue cytokine and immune cell distribution was determined using immunohistochemistry. Systemic cytokine production was evaluated by multiplex-cytokine assays.

Results Iron expression was distributed to the epithelium of tumour tissues in OI treatment $(\mathrm{p}<0.001)$ and to the tumour stroma and the macrophages in IVI treatment $(p<0.01)$. OI treatment significantly increased the production of the systemic pro-inflammatory cytokines; IL-6 $(\mathrm{p}<0.02)$, IL-12 $(\mathrm{p}<0.03)$, IL-17 $(p<0.04)$, GM-CSF $(p<0.02)$ and IFN- $\gamma \quad(p<0.03)$. Moreover, OI treatment decreased the anti-inflammatory cytokine IL-4 $(\mathrm{p}<0.01)$ and IVI increased the anti-inflammatory cytokine IL-10 $(\mathrm{p}<0.003)$ production in the serum. Immunoreactivity of IL-12 levels was higher in tissues from patients treated with OI and distributed to the epithelium. However, there were no significant differences in immunoreactivity of tissue IL-10 between the treatment groups. Inflammatory cell (lymphocytes and granulocytes) infiltration was higher in the OI treatment group.

Conclusions Intravenous iron is compartmentalised to non-epithelial cells indicating that iron is potentially less bio-available to the tumour cells. Oral iron treatment induces inflammatory immune responses both systemically and within the tumour microenvironment. Further functional studies are ongoing to evaluate the mucosal immune response to differential iron loading.

\section{REFERENCES}

1. Bergman $M$, et al., Biomed Pharmacother. 2005;59(6):307.

2. Glei M, et al., Mutat Res. 2002 26:519(-):151. 